\title{
Study on Three-Dimensional Teaching Reform of Mechanical Engineering Subject Based on the Cultivation of Innovative Ability Hongjun Yang ${ }^{1, a,{ }^{*}}$ \\ ${ }^{1}$ School of Mechanical Engineering, Wuhan Polytechnic University, Wuhan, Hubei, China \\ a hongjun_yang@163.com \\ ${ }^{*}$ Corresponding author
}

\begin{abstract}
Aiming at the shortage of the traditional teaching mode in mechanical engineering subject, the three-dimensional teaching reform was put forward based on the cultivation of innovative ability. The principles of construction of three-dimensional teaching resources were considered and the system structure was brought according to the three-dimensional teaching objectives. Then the three-dimensional teaching methods were discussed in detail from four aspects, including teaching content, teaching mode, learning mode and teaching evaluation. Using this teaching reform, the teaching instruction function of the teachers and activeness of the students can be increased greatly, which helps to cultivate students' innovation ability.
\end{abstract}

Keywords: Three-dimensional teaching, Mechanical engineering subject, Innovation ability

\section{基于创新能力培养的机械工程学科立体化教学改革的研究 \\ 杨红军 ${ }^{1, a,{ }^{*}}$ \\ 1 武汉轻工大学机械工程学院, 武汉, 湖北, 中国 \\ a Hongjun_yang@163.com \\ *通讯作者}

中文摘要. 针对机械工程学科的传统教学 方式的不足, 本文提出了基于创新能力培养 的立体化教学改革的观点。在充分考虑立体 化教学目标的基础上, 提出了立体化教学资 源的建设原则, 并给出了立体化教学资源的 体系结构。最后, 从四个方面详细讨论实施 立体化教学的具体方法, 包括教学内容、教 学模式、学习模式和教学评价。利用立体化 教学的方法, 可以充分发挥教师教学的指导 作用, 提高学生学习的主动性, 从而有利于 培养学生的创新能力。
关键词: 立体化教学; 机械工程学科; 创新 能力

\section{1. 引言}

21 世纪是知识和科技创新的时代, 创新 型人才的培养直接关系到一个国家的科技 进步和社会的发展, 关系到国家竞争力和综 合实力的提升。高校, 特别是理工科院校, 作为科技类人才培养的重要基地, 其培养质 量的高低对于我国的科技竞争力起着决定 
性的作用。机械工程学科是培养制造业人才 的一门主要学科, 是关系国民经济支柱产业 的一门学科, 对创新型人才的培养提出了更 高的要求。传统的教学方式由于教学手段单 一、方法固定、效率低下等不足, 已不能很 好地满足当前的教学需要。

为提高教学质量和效果, 各高等院校正 进行着教学改革, 各种新的教育理念、方法 与技术不断涌现。其中以现代信息技术为支 撑, 以立体化教学资源建设为基础, 强调教 学活动过程中人、物、环境相协调的多维的、 多层次的立体化教学方式, 是当前教学改革 的重要方向之一。立体化教学的概念最早出 现于立体化教材的建设中, 2003年4月, 教 育部在立体化教学精品课程建设的通知中, 首次提出立体化教材应以电子及网络存储 为载体, 以网络为传输手段的新型出版物, 包括电子教案、电子图书、CAI课件、试题 库、网络课程和资料库等六类 ${ }^{[1]}$ 。随后, 从 立体化教材的概念发展到了立体化教学资 源的概念, 高等教育出版社在 “教学资源立 体化建设计划” 中提出了立体化教学资源包 括教学包、教学资源库、教学网站建设三个 方面 ${ }^{[2]}$ 。空军工程大学的宋云娴教授认为立 体化教学资源是以现代化的信息技术为手 段, 以适合远程传输的数字化教育教学软件 为教材, 以Internet / Intranet为学习和管理 环境, 以自主式、开放式、交互式学习为主 体学习模式, 以媒体素材为基础, 适用于多 层次教学对象, 覆盖教学的全过程各个环节 而构建的教学资源体系, 包括教学信息系统 化, 教学环境数字化, 教学对象层次化三个 方面的内涵 ${ }^{[3]}$ 。华东师范大学的余朝文指出 立体化教育资源应从 “人”、“物”、“环 境” 三个维度来探讨, 以计算机网络为基本 教学和管理环境, 以现代信息技术为手段, 以适合远程网络传输的数字化教育教学信 息为载体, 以人为本, 帮助以自主式、开放 式、交互式学习为主体的学习模式进行学 习, 服务于所有成人对象在整个教学过程中 各个环节的教学资源 ${ }^{[4]}$ 。沈阳师范大学的宋 倬指出新模式的高校教学资源应该是教学 包、教学资源库和学科 (专业) 网站的有机 结合体, 并具有开放性、实践性、互动性、 灵活性等特点 ${ }^{[5]}$ 。对机械工程学科实施立体
化教学改革, 就必须首先建设好立体化教学 资源, 并根据立体化教学资料在实际教学中 进行立体化教学模式的教学改革, 才能达到 事半功倍的效果, 提高教学的质量, 培养创 新型的人才。

\section{2. 立体化教学资源的建设}

\section{1 立体化教学资源建设的指导思想}

机械工程学科是典型工科学科, 其专业 课的设置涉及到数学、力学、电子、光学、 控制、计算机、先进制造等复杂抽象的内容。 由于课程繁多, 学时有限, 内容又相对抽象, 如果仅凭课程讲授, 往往达不到很好的效 果, 学生对知识的掌握也只是一知半解。如 果充分利用现代信息技术, 大力开发立体化 教育资源, 推进立体化教育模式的改革, 对 促进教学质量, 培养创新能力是非常有帮助 的。立体化教学资源的建设必须遵循一定的 指导思想。

\subsection{1坚持创新型人才培养的原则}

立体化教学目的在于培养创新型人才, 所以资源的建设并不各种纸质或电子版资 料的堆积, 必须注重资源对于创新型人才培 养的作用。资源的选取上应该有助于进行立 体化教学改革, 例如, 教材的建设上不再是 强调知识的堆积, 而是重逻辑推理及知识间 的联系和思维引导; 案例素材的选取注重对 学生的创新思维的培养, 能够适用于问题式 或研究式教学; 多媒体课件的制作上强调趣 味性及知识性并重、注重培养学生的逻辑思 维, 能够引导学生进行问题讨论; 实践教学 材料选取上注重对学生动手能力的培养, 给 学生更多的思考空间, 等等。

\subsection{2坚持开放性的原则}

立体化教学资源必须坚持开放性, 这里 的开放性是指面向教师及学生开放两个方 面。教师应该能够根据立体化教学的需要在 教学过程随时获取教学资源, 另外教师作为 资源库的管理和维护者, 还应该能够随时在 资源库中增加新内容, 以做到知识的更新, 这类似于专家系统中的知识库。另外教学资 源还要面向学生开放, 学生应该能够直接利 用教学资源进行自主学习, 包括理论学习、 自我测练、与教师及同学进行交流和讨论 
等, 并且可以制定学习计划并保存学习的进 度。

\subsection{3坚持先进性的原则}

立体化教学资源的建设是以信息技术 为基础的, 所以必须充分利用目前先进的信 息科学技术来多方位、立体化建设教学资 料。一方面, 充分利用基于网络的现代信息 处理技术，包括面向对象的网络编程技术、 多媒体及网页制作技术、数据库技术等, 保 证教学资源的先进性及安全性。另一方面, 从适应立体化教学改革的角度出发, 充分利 用BBS、微博、微信等技术, 开展基于网络 的立体化教学, 包括网上远程授课、网上测 试、网上讨论、网上答疑等。

\section{2 立体化教学资源的组成}

立体化教学资源是多层次、多方面的。 目前, 在大学英语、计算机教学等学科门类 中, 立体化的教学资源比较丰富, 但对于机 械工程学科等传统工科来说, 则进展较为缓 慢, 主要表现在: 对立体化教学的内涵认识 不足, 在教学资源建设上只是简单地重复前 人的工作, 缺乏创新, 内容上尚处于较低的 层次, 有些只是停留在多媒体课件的阶段, 有些虽然有教学网站, 但缺乏实时的互动交 流, 留于形式, 对教学的辅助效果不强。立 体化教学资源应该包括立体化教学包、立体 化教学资源库、立体化教学网站三个层次, 这三个层次包含在立体化教学的三个维度 上, 如图1所示。其中, 理论教学资源主是 指为课堂教学服务的资源, 包括纸质的立体 化教材、教学参考书, 电子化的多媒体课件、 网络化的课堂教学等。而实践教学是指工程 实践、实训、实验等各种资源, 包括各种虚 拟仿真实验等。辅助教学则是指课后的教学 辅导及学生自学等各种资源, 包括教学网站 上的学习资源等。

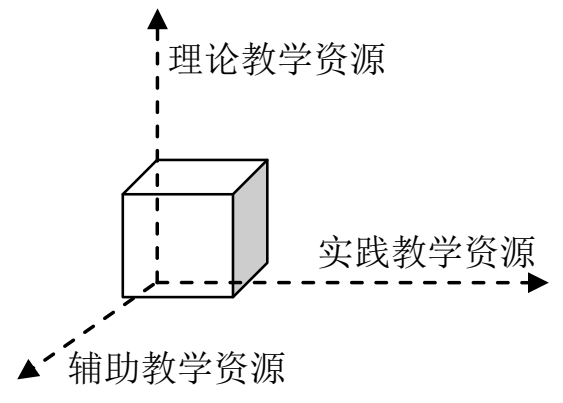

图1 立体化教学资源库的三维结构

\subsection{1 立体化教学包的建设}

立体化教学包是立体化资料是第一个 层次, 包括主教材、教学参考书、学习指导 书、习题集、试题库、电子教案、实验实训 教材、实验指导书、视频动画库等。根据机 械工程的学科特点, 应该认真编写好各门课 程的教学大纲, 选择合适的教材, 或直接编 写相关教材及参考书, 设计相应的课程实 验, 认真编写实验指导书; 充分利用多媒体 技术制作形式活泼、内容多样、适合立体化 教学的多媒体课件; 收集编写及大量习题, 建立习题库及试题库, 为立体化教学的资源 库的建立作好相应的准备。立体化教学包将 教学的内容以立体化的、不同形态的方式呈 现出来, 可以直接在在教学中发挥各自的优 势, 满足多种不同应用的需要。

\subsection{2 立体化教学资源库的建设}

立体化教学资源库是立体化教学资源 建设的第二个层次, 是指利用计算机软件编 程及数据库管理技术, 将立体化教学包的内 容按照一定的组合方式, 分别加入到所设计 的各个不同的功能模块中。立体化教学资源 库主要包括系统管理模块、理论教学部分模 块、实践教学模块及教学辅助模块共四个模 块。图2给出了立体化教学的资源库的结构。 管理模块处于最顶层, 可以对其余三个模块 进行管理，包括系统信息、用户管理 、数 据库管理等, 用于对系统的用户注册、权限、 帐号管理, 以及对系统数据库进行更新、备 份等操作。其余三个模块则处于并行地位, 分别适用不同的教学形式。

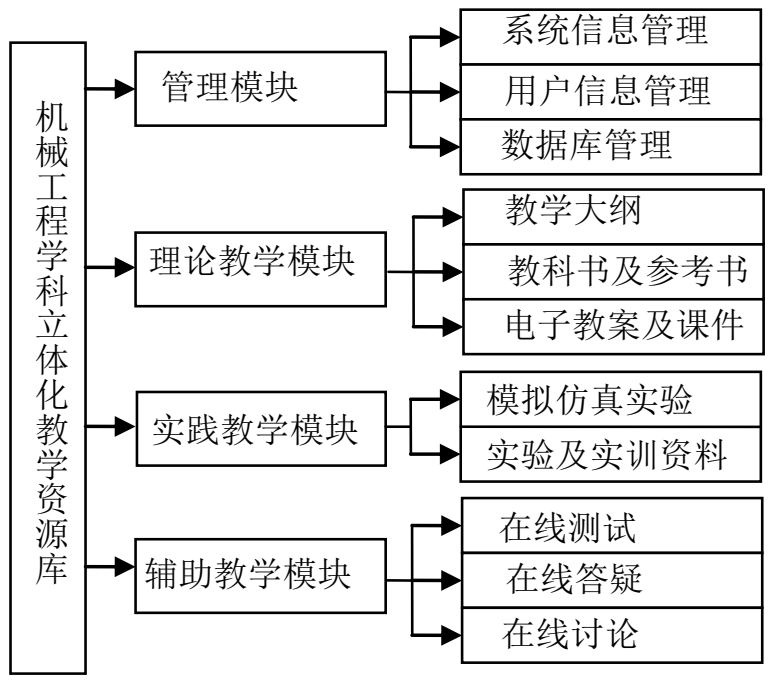

图2. 立体化教学资源库的组成 


\subsection{3立体化教学网站的建设}

立体化教学改革是以信息技术为基础 的, 而网络技术是信息技术的重要方面。立 体化教学模式的实现, 必须以现代计算机网 络为载体, 开发立体化教学资源, 通过教与 学的网络互动, 从而达到教学多样化、立体 化的目的。立体化教学网站是立体化教学资 源建设的第三个层次, 是为立体化教学服务 的综合性的平台。教师可通过教学网站进行 远程网络授课, 对学生进行在线辅导; 学生 可通过教学网站进行自主学习及自我测试, 并通过讨论区、微博、微信及QQ等各种模 式参与课程的讨论及学习, 形成师生互动、 学生之间互动等。网站可提供丰富的教学资 源, 包括电子课件、音像、动画等, 并且提 供在线习题练习、在线考试、在线模拟仿真 实验各种功能模块, 满足各种不同水平的学 生的学习的需要。教学网站实际上立体化教 学包及立体化教学资源库的具体应用, 是综 合性程度最高立体化教学资源。

\section{3. 立体化教学的实施}

\section{1 教学内容的立体化改革}

无论在任何情况下, 理论教学在教学中 的总是处于第一位的, 理论教学承担着传授 知识的重任, 也承担着培养创新能力的重 任, 但传统的理论教学方式只重知识的灌 输, 学生处于被动接受状态, 效果不太理想。 立体化教学改革就是要在教学资源配置、教 学模式上进行改革, 适应学生创新能力培养 的需要。机械工程学科是一门古老的学科, 同时又是一门不断发展的综合性很强的学 科, 牵涉到的力学、电学、数学、计算机等 多门学科。其课程设置十分庞杂, 但有些课 程的知识点重复率很多, 有些课程的关联性 非常强, 因而需要大力进行相关的课程建 设, 将部分课程进行整合, 精炼课程的内容, 进行必要的知识更新, 查漏补缺, 优化课程 体系及教学的内容。具体对于某一门课程而 言, 首先应以培养学生创新思维能力为指导 思想, 重新编写课程教学大纲, 对课程的教 学内容进行优化, 强调理论讲授、学生自学、 课程讨论、课程课外论文或作品制作等各部 分内容的分配比例。此外, 还必需补充教学
参考书、习题集、辅导书、电子多媒体课件, 音视频资料等, 从而形成立体化的教学内 容。

\section{2 教学模式的立体化改革}

有研究指出, 一个本科生毕业在校所学 的知识, 仅占其一生所需知识的 $10 \%$, 其余 90\%需要在以后的工作中来获得。高校承担 着培养高级人才的任务, 但不应该只是知识 的灌输者, 而是应该教会学生如何去领悟掌 握更多的知识, 所谓 “授人以鱼, 不如授人 以渔”, 即为此道理。要培养创新型的人才, 就必须对传统的教学方法进行改革, 采用问 题式教学、研讨式教学、案例式教学、工程 实践式教学等各种不同的立体化的教学方 法。问题式教学是创造一种问题的情境, 并 提出相应的要解决的问题, 然后引导学生分 析与思考问题，进行相关科技资料的收集与 归纳, 探求解决问题的方法, 并得出科学的 结论, 获取新的知识。研讨式教学是教师就 某一知识重点和难点设计相应的题目, 然后 将学生分成若干组, 每小组就这一问题进行 分析讨论, 分工协作, 探求解决方法, 从而 激活学生的思维, 深化学生对知识点的理解 和运用。案例式教学是教师通过典型的工程 实例, 采取循序渐进, 逐步深入的方法对学 生进行讲解, 注意对学生进行提问与诱导, 最后给出工程问题的最终解决方案, 从而加 深学生对重要理论知识的理解, 并了解工程 设计的基本步骤。工程实践式教学是让学生 直接参与教师科研课题或各种层次科技制 作或比赛, 培养其动手能力和创新能力, 从 而增强对本学科的理解与认识。

\section{3 学习模式的立体化改革}

传统的学习模式是教师理论传输及学 生课下复习为主, 学生的学习主动性及积极 性不高。立体化教学改革必须改变这种的固 定的、效率低下的学习模式, 培养形式多样 的立体化的学习模式, 从而培养学生的创新 能力。因而, 首先必须提供一个立体化的学 习环境, 一方面, 在课堂上, 由教师采用立 体化的教学模式, 包括问题式、研讨式等各 种研究性教学方法的运用, 让学生沉浸在一 个丰富多彩、富有探索的学习空间中。另一 方面, 通过教学网站等教学资源, 给学生提 
供一种虚拟的、自由探索的学习环境, 让学 生能够利用各种网络工具和资源来进行学 习, 并在学习过程通过网络交互, 得到来自 老师和同学的帮助和支持, 达到学习的目 的。学生能够根据教学网站所提供的教学资 源, 利用立体化的手段, 不受时空的限制, 根据自身对教学内容的理解情况, 合理地选 择学习内容和学习方法, 安排学习的时间和 进度, 并能完成测试考核, 检查学习的效果。 由于网络提供了图文并茂、音响动画、视频 资料等丰富多彩的内容, 激发了学生的学习 兴趣, 创建了学习的情境, 从而使学生的学 习变为自主学习行为, 培养了学生的创新能 力。

\section{4 教学评价方式的立体化}

传统的教学评价方式主要是通过试卷 考核的方式来评定学生的学习成绩, 一考定 论、甚至一考定终生, 这种评价方式的弊端 是不能全面地考核一个学生的综合能力, 容 易培养一些考试机器。很多学生为了应付考 试往往平时不努力, 考前通过投机复习, 甚 至一些不端的行为来获得成绩, 但真正对该 门课程的掌握却十分肤浅, 甚至考后就忘, 更谈不上创新思维和能力。

进行立体化教学的改革, 就必须改革传 统的教学评价方式, 采用多样的、立体化的 课程成绩评价方式。因此, 需要根据课程特 点制定各种评价指标及其权重, 合理评价学 生的成绩, 这包括: 课程测验、课外科技制 作或论文、课程讨论及总结资料、课堂作业 及考勤、期中期末考试等。

\section{4. 结束语}

机械工程学科作为一门主要工程学科, 担负着培养国家创新型人才的重任。传统的 教学方法由于效率低下、不适应新时代人才 培养需要等弊端必须进行一定的改革, 以现 代信息技术为支撑的新的立体化教学方法 可以很好解决这些问题。此外, 这种教学方 法还能够解决有关课程教学学时偏短、实验
硬件资源不足等缺点, 实现真正意义上的教 与学的互动。本文所提出的教学资源的构建 原则及教学资源的具体内容可以有效地为 立体化教学的实施提供基础支持, 而所提出 的立体化的教学实施办法全面包括了教学 内容、教学理念、学习模式、教学评价等各 个方面, 从近年来在本校有关课程的实施结 果来看, 取得了较好的效果。

\section{致谢}

本文的研究得到湖北省教育科学“十二 五” 规划课题(2014B182)及武汉轻工大学教 学研究项目(XZ2015015)的支持, 在此表示 感谢。

\section{References}

[1] Ministry of Education, China. Notice of Quality Course Construction in Teaching Quality and Reform Engineering[R]. Beijing, China, 2003.

[2] Hong Wang. Positioning and System Structure of Three-Dimensional Teaching Resources [M]. Higher Education Press, Beijing, China, 2004.

[3] Yunxian Song, Fanshi Han and Jun Wu. Construction of Three-dimensional Model of Teaching Resources[J]. Journal of Electrical \& Electronic Engineering Education, 2005, 27(2): 100-103

[4] Zhaowen Yu. Constuction Scheme of Three-Dimensional Teaching Resources on Web Based Adult Education [D]. East China Normal University, Shanghai, China, 2007.

[5] Zhuo Song. Research on construction of Three-Dimensional teaching resources of VFP course [D]. Shenyang Normal University, Shenyang, China, 2008. 